\title{
Pseudomonas Aeruginosa: Freqüência de Resistência a Múltiplos Fármacos e Resistência Cruzada entre Antimicrobianos no Recife/PE*
}

\section{Pseudomonas Aeruginosa: Frequency of Resistance to Multiple Drugs and Cross-Resistance between Antimicrobials in Recife/PE}

\author{
Eduardo Andrada Pessoa de Figueiredo', Heloisa Ramos², Maria Amélia Vieira Maciel${ }^{3}$, \\ Maria do Carmo Monteiro Vilar ${ }^{4}$, Noel Gomes Loureiro ${ }^{5}$, Rodrigo Gomes Pereira ${ }^{6}$
}

\section{RESUMO}

JUSTIFICATIVA E OBJETIVOS: A multi-resistência bacteriana tem crescido significativamente nos últimos anos. Entre os gram-negativos a $P$. aeruginosa demonstra maior facilidade de desenvolvimento de resistência aos antibióticos. O objetivo deste estudo foi determinar os padrões de susceptibilidade antimicrobiana, freqüência de resistência a múltiplos fármacos e de resistência cruzada entre antimicrobianos das cepas de Pseudomonas aeruginosa.

MÉTODO: O estudo foi realizado entre setembro de 2004 e janeiro de 2006. Os testes de susceptibilidade antimicrobiana foram realizados em 304 cepas de $P$.

1. Mestre pelo Programa de Pós-Graduação em Ciências da Saúde da UFPE. Especialista em Medicina Interna pela UFPE.

2. Professora Doutora Adjunta do Departamento de Medicina Clínica, CCS-UFPE e da UPE; Vice-Coordenadora da UTI do Hospital Esperança, Recife.

3. Professora Assistente do Departamento de Microbiologia da UFPE.

4. Técnica do Laboratório de Microbiologia do Hospital das Clínicas da UFPE.

5. Residente do Programa em Clínica Médica do Hospital Agamenon Magalhães do Sistema Único de Saúde/PE.

6. Graduando do Curso de Medicina da UFPE.

*Recebido do Curso de Pós-Graduação em Ciências da Saúde da Universidade Federal de Pernambuco (UFPE), Recife, PE.

Apresentado em 17 de julho de 2007

Aceito para publicação em 02 de outubro de 2007

Endereço para correspondência:

Dr. Eduardo Andrada Pessoa de Figueiredo

Rua Setúbal, 566/301 -- Boa Viagem

51030-010 Recife, PE

Fones: (81) 33413689 - 9954-9549

E-mail: eduardof@hotlink.com.br

(C)Associação de Medicina Intensiva Brasileira, 2007 aeruginosa segundo os padrões do National Committee for Clinical and Laboratory Standards (NCCLS).

RESULTADOS: Os materiais mais freqüentes foram urina com $26,7 \%$ e secreção traqueal com $26,1 \%$. Os seguintes antibióticos, com respectivos percentuais de susceptibilidade, foram observados: piperacilinatazobactam (66,2\%); aztreonam (59,8\%); amicacina $(59,4 \%)$; meropenem $(58,2 \%)$; imipenem $(57,7 \%)$; ciprofloxacina $(49,7 \%)$; gentamicina e cefepima $(48,6 \%)$; ceftazidima $(30 \%)$ e cefotaxima $(6,8 \%)$. Detectou-se elevada prevalência de multi-resistência, com 49,7\% das cepas resistentes a três antibióticos ou mais e $28 \%$ resistentes a seis antibióticos ou mais. Adicionalmente se demonstraram taxas de resistência cruzada entre os beta-lactâmicos (carbapenêmicos e piperacilina/tazobactam) e os aminoglicosídeos e quinolonas entre $22,9 \%$ e $38,1 \%$ (fármacos comumente utilizados como adjuvantes no tratamento das infecções graves por pseudomonas), refletindo dificuldade nas opções de associação de antimicrobianos para tratamentos combinados.

CONCLUSÕES: A freqüência de cepas multi-resistentes de $P$. aeruginosa foi semelhante à descrita na literatura nacional e maior do que a mundial. Para reduzir a freqüência destes clones multi-resistentes, monitorização epidemiológica e racionalização de antimicrobianos devem ser implementadas urgentemente.

Unitermos: epidemiologia, multi-resistência, Pseudomonas aeruginosa

\section{SUMMARY}

BACKGROUND AND OBJECTIVES: The frequency of multiple-antibiotic resistant bacteria has been increasing in recent years. Among the gram-negative bacteria Pseudomonas aeruginosa ( $P$. aeruginosa) shows a great 
propensity for the development of multidrug resistance mechanisms. The objective of this study was to identify the profile of susceptibility to antibiotics, the frequency of multidrug resistance and the cross-resistance between drugs of $P$. aeruginosa strains in two tertiary hospitals in Recife, Pernambuco.

METHODS: The study was carried out between September 2004 and January 2006. The antimicrobial susceptibility testing was performed in 304 strains of $P$. aeruginosa by the disc diffusion method in accordance with National Committee for Clinical and Laboratory Standards (NCCLS) guidelines.

RESULTS: The most frequent materials were urine $(26.7 \%)$ and respiratory tract secretion $(26.1 \%)$ The antibiotics tested and their respective susceptibilities were as follows: piperacillin-tazobactam (66.2\%); aztreonam (59.8\%); amikacin (59.4\%); meropenem (58.2\%); imipenem (57.7\%); ciprofloxacin (49.7\%); gentamicin and cefepime (48.6\%); ceftazidime (30\%) and cefotaxime (6.8\%). A high prevalence of multi-resistance was detected. Half $(49.7 \%)$ the strains showed resistance to three or more antibiotics and $28 \%$ were resistant to six antimicrobials or more. Also, cross-resistance between the beta-lactams (carbapenems and piperacilin/tazobactam) and aminoglicosides and quinolones was between $22.9 \%$ and $38.1 \%$. These drugs are commonly combined in the treatment of severe infections caused by Pseudomonas, which reflects the difficulty in choosing the appropriate option for combination therapy.

CONCLUSIONS: The frequency of multidrug-resistant strains of $P$. aeruginosa in this study was similar to other hospitals in Brazil and higher than in other countries. In order to reduce the frequency of these multiresistant clones, epidemiologic surveillance and the rational use of antibiotic protocols need to be urgently implemented.

Key Words: antimicrobial susceptibility, multidrug-resistance, Pseudomonas aeruginosa.

\section{INTRODUÇÃO}

A Pseudomonas aeruginosa ( $P$. aeruginosa) pode causar infecções nosocomiais graves, com elevada letalidade $^{1-3}$. Atualmente se posiciona entre as principais bactérias causadoras de infecções hospitalares, perdendo apenas para o Staphylococcus coagulase negativo e o Staphylococcus aureus ${ }^{4}$. Relatos de redução da susceptibilidade da $P$. aeruginosa aos antimicrobianos vêm sendo publicados no Brasil $^{5-7}$ e em outros países $^{8-14}$ destacando-se a diminuição de sensibilidade aos antibióticos de maior espectro de ação como os carbapenêmicos e as cefalosporinas anti-pseudomonas $^{12,13,15}$. Entre as mutações que acarretam em aumento da resistência, a produção de enzimas betalactamases e metallo-beta-lactamase ${ }^{16-19}$ são as de maior importância e geralmente ocorrem em pacientes com maior tempo internação e uso prévio de antimicrobianos $^{11,20}$.

Outra característica marcante e preocupante desta espécie, é a resistência cruzada aos antimicrobianos, que resulta da co-resistência, ou seja, da presença de múltiplos mecanismos de resistência num único hospedeiro levando à resistência a múltiplos fármacos ${ }^{11,17}$. No Brasil, particularmente na região Nordeste, poucos são os estudos que descrevem o perfil fenotípico das bactérias Gram-negativas em especial a $P$. aeruginosa ${ }^{21}$. O objetivo deste estudo foi determinar os padrões de susceptibilidade antimicrobiana, a freqüência de resistência a múltiplos fármacos e de resistência cruzada entre antimicrobianos das cepas de $P$. aeruginosa de dois hospitais terciários.

\section{MÉTODO}

Foram estudadas 191 culturas positivas para $P$. aeruginosa oriundas do Hospital das Clínicas da Universidade Federal de Pernambuco (HC-UFPE), obtidas consecutivamente no período de setembro de 2004 à agosto de 2005 e 113 cepas oriundas do Hospital Agamenon Magalhães, ligado à Secretaria Estadual de Saúde (SUS), obtidas no período de março de 2005 a janeiro de 2006. As 304 amostras foram provenientes de todas as unidades (UTI e outras alas, excetuando-se a pediátrica) de ambos os hospitais e incluíam sangue, urina, secreção traqueal, secreção de ferida operatória, úlcera de pele, ponta de cateter, dentre outros. Os dados referentes à identificação bacteriana e aos testes de sensibilidade foram obtidos nos registros dos laboratórios de análises clínicas dos respectivos hospitais. Foi considerada apenas uma cultura por paciente (a primeira cultura registrada no laboratório). A $P$. aeruginosa foi identificada pela morfologia da colônia, prova de oxidase positiva, produção de piocianina em meio agar Mueller-Hinton (BIOBRAS S.A), motilidade e crescimento em meio centrimida Agar. Para a realização dos antibiogramas, antes da colocação dos discos, as placas de Mueller-Hinton foram inoculadas com swabs submersos na solução final de inoculação e repassados sobre toda a superfície da placa. As placas foram incubadas aerobicamente por 18 a 24h, com tempera- 
tura de $35^{\circ}-37^{\circ} \mathrm{C}$. Os testes de sensibilidade utilizaram as seguintes concentrações de antimicrobianos: amicacina $(30 \mu \mathrm{g})$, aztreonam $(30 \mu \mathrm{g})$, ceftazidima $(30 \mu \mathrm{g})$, ceftriaxona $(30 \mu \mathrm{g})$, cefotaxima $(30 \mu \mathrm{g})$, ciprofloxacina $(5 \mu \mathrm{g})$, gentamicina $(10 \mu \mathrm{g})$, cefepima $(30 \mu \mathrm{g})$, imipenem $(10 \mu \mathrm{g})$, meropenem $(10 \mu \mathrm{g})$, piperacilina/tazobactam $(100 / 10 \mu \mathrm{g})$ (esse último antimicrobiano foi observado somente nas amostras do HAM). A sensibilidade foi investigada utilizando o método de Kirby-Bauer para leitura dos discos de difusão, de acordo com os critérios do National Committee for Clinical and Laboratory Standards (NCCLS) ${ }^{22,23}$. O teste de sensibilidade à polimixina não foi realizado por não estar adequadamente padronizado durante a realização do estudo.

Para análise dos dados foram obtidas distribuições absolutas e percentuais uni e bivariados (técnicas de estatística descritiva) e foi utilizado o teste Qui-quadrado de Pearson ou o teste Exato de Fisher quando as condições para utilização do Qui-quadrado não foram verificadas ${ }^{24,25}$. Os dados foram digitados na planilha Excel e o programa estatístico utilizado para a obtenção dos cálculos estatísticos foi Statistical Analysis System (SAS) 8.06

\section{RESULTADOS}

Dentre as 304 cepas analisadas $81(26,7 \%)$ foram isoladas da urina; $79(26,1 \%)$ da secreção traqueal; 60 $(19,8 \%)$ da pele, partes moles e feridas; $26(8,6 \%)$ da ponta de cateter, $12(4 \%)$ do sangue e 45 (14,8\%) de outros materiais.

Do total de cepas isoladas 79 (26\%) foram provenien- tes de UTI e $154(50,7 \%)$ foram coletadas em outras alas dos hospitais. Em 71 amostras $(23,3 \%)$ não foi possível identificar o local da coleta.

A tabela 1 mostra os resultados dos testes de sensibilidade obtidos das 304 cepas de $P$. aeruginosa de acordo com o local de origem. O composto mais ativo contra a $P$. aeruginosa no HAM foi a piperacilina-tazobactam $(66,1 \%)$. No HC os compostos mais ativos foram os cabapenêmicos (imipenem com $62,7 \%$ de susceptibilidade seguida pelo meropenem com $62,1 \%$ de susceptibilidade). A sensibilidade global das cepas de $P$. aeruginosa, com relação aos antimicrobianos estão apresentadas na tabela 1. Diferença estatisticamente significativa $(p<0,05)$ foi encontrada na análise comparativa entre os dois hospitais, com maior susceptibilidade das Pseudomonas isoladas no $\mathrm{HC}$ aos antibióticos cefepima ( $p<0,0001)$, ciprofloxacina $(p<0,0034)$, gentamicina $(p<0,0001)$ e imipenem $(p<0,0384)$.

Das cefalosporinas observadas, o cefepima foi a que mais reteve atividade contra a $P$. aeruginosa nos dois hospitais, com $58,6 \%$ das cepas do $\mathrm{HC}$ e $32 \%$ do HAM sensíveis e sensibilidade global de $48,6 \%$ nos dois hospitais, enquanto que a ceftazidima apresentou sensibilidade em apenas $30 \%$ das amostras dos dois hospitais.

Os carbapenêmicos mais ativos foram o meropenem $(58,2 \%)$ seguido do imipenem $(57,7 \%)$, na avaliação dos dois hospitais.

As taxas de resistência à ciprofloxacina foram altas, apenas $49,7 \%$ de todas as cepas apresentaram atividade contra a $P$. aeruginosa. Dentre os aminoglicosídeos, a susceptibilidade foi de $59,4 \%$ para a amicacina

Tabela 1 - Sensibilidade da P. Aeruginosa, por Antibiótico nos Hospitais Estudados

\begin{tabular}{|c|c|c|c|c|c|c|c|}
\hline \multirow{3}{*}{ Antibióticos } & \multicolumn{6}{|c|}{ Hospitais } & \multirow{3}{*}{ Valor de $p$} \\
\hline & \multicolumn{2}{|c|}{$\mathrm{HC}$} & \multicolumn{2}{|c|}{ Agamenon Magalhães } & \multicolumn{2}{|c|}{ Os Dois Hospitais } & \\
\hline & $\begin{array}{l}\text { Sensíveis } \\
\text { (Testadas) }\end{array}$ & $\begin{array}{c}\% \text { de } \\
\text { Sensíveis }\end{array}$ & $\begin{array}{l}\text { Sensíveis } \\
\text { (Testadas) }\end{array}$ & $\begin{array}{c}\% \text { de } \\
\text { Sensíveis }\end{array}$ & $\begin{array}{l}\text { Sensíveis } \\
\text { (Testadas) }\end{array}$ & $\begin{array}{c}\% \text { de } \\
\text { Sensíveis }\end{array}$ & \\
\hline Amicacina & $98(159)$ & 61,6 & $63(112)$ & 56,3 & $161(271)$ & 59,4 & $p^{1}=0,3740$ \\
\hline Aztreonam & $106(106)$ & 61,3 & $62(108)$ & 57,4 & $168(281)$ & 59,8 & $p^{1}=0,5205$ \\
\hline Cefepima & 95 (162) & 58,6 & $31(97)$ & 32 & $126(259)$ & 48,6 & $\mathrm{p}^{1}<0,0001^{*}$ \\
\hline Cefotaxima & $18(171)$ & 10,5 & $8(95)$ & 8,4 & $26(266)$ & 9,8 & $\mathrm{p}^{1}=0,5796$ \\
\hline Ciprofloxacina & $101(179)$ & 56,4 & $43(38,7)$ & 38,7 & $144(290)$ & 49,7 & $p^{1}=0,0034^{*}$ \\
\hline Gentamicina & $106(185)$ & 57,3 & 35 (105) & 33,3 & $141(291)$ & 48,6 & $\mathrm{p}^{1}<0,0001^{*}$ \\
\hline Imipenem & $104(166)$ & 62,7 & $54(108)$ & 50 & $158(274)$ & 57,7 & $\mathrm{p}^{1}=0,0384^{*}$ \\
\hline Meropenem & $59(95)$ & 62,1 & $55(101)$ & 54,5 & $114(196)$ & 58,2 & $p^{1}=0,2779$ \\
\hline Piperacilina-tazobactam & NT & & 72 (109) & 66,1 & - & - & - \\
\hline Ceftazidima & NT & & 27 (89) & 30,3 & - & - & - \\
\hline
\end{tabular}

NT = Não testada

* - Associação significativa ao nível de 5\%.

1 -Teste Qui-quadrado de Pearson.

Os valores entre parêntesis representam o total de amostras testadas. 
e de $48,6 \%$ para a gentamicina.

A tabela 2 mostra uma análise comparativa da sensibilidade das cepas de $P$. aeruginosa por local da coleta das amostras (cepas oriundas das UTI comparadas às cepas oriundas de outros locais dos hospitais) em cada um dos hospitais. Para todas as cepas observadas no HAM, as diferenças existentes entre as sensibilidades não foram estatisticamente significativas ( $p$ $<0,05$ ). Para as cepas estudadas no $\mathrm{HC}$, a diferença de susceptibilidade foi estatisticamente significativa para os antibióticos: amicacina, aztreonam, cefepima, ciprofloxacina, gentamicina e imipenem.

A tabela 3 apresenta uma análise em percentual da resistência cruzada entre a amicacina e os antibióticos imipenem e meropenem nos dois hospitais, adicionalmente com a piperacilina-tazobactam no HAM. As taxas de resistência cruzada foram: para o imipenem $30,6 \%$ no $\mathrm{HC}, 35,2 \%$ no HAM e $32,4 \%$ em ambos os hospitais; para o meropenem $30,7 \%$ no $\mathrm{HC}, 35,6 \%$ no HAM e 33,3\% em ambos os hospitais. No HAM, para a piperacilina-tazobactam $22,9 \%$. As taxas de resistência cruzada da ciprofloxacina foram: para o imipenem

Tabela 2 - Sensibilidade da P. Aeruginosa, por Antibiótico, segundo a Unidade de Coleta nos Hospitais Estudados

\begin{tabular}{|c|c|c|c|c|c|c|c|c|}
\hline \multirow{3}{*}{ Antibióticos } & \multirow{3}{*}{ Hospital } & \multicolumn{4}{|c|}{ Local de Coleta } & \multirow{2}{*}{\multicolumn{2}{|c|}{ Total das coletas }} & \multirow{3}{*}{ Valor de $p$} \\
\hline & & \multicolumn{2}{|c|}{ UTI } & \multicolumn{2}{|c|}{ Não UTI } & & & \\
\hline & & $\mathrm{N}$ & $\%$ & $\mathrm{~N}$ & $\%$ & $\mathrm{n}$ & $\%$ & \\
\hline \multirow[t]{2}{*}{ Amicacina } & $\mathrm{HC}$ & $15(48)$ & 31,3 & $46(61)$ & 75,4 & $61(109)$ & 56 & $\mathrm{p}^{1}<0,0001^{*}$ \\
\hline & Agamenon Magalhães & $16(27)$ & 59,3 & 47 (84) & 54,8 & 66 (111) & 55,9 & $\mathrm{p}^{1}=0,6822$ \\
\hline \multirow[t]{2}{*}{ Aztreonam } & $\mathrm{HC}$ & $21(52)$ & 40,4 & $46(68)$ & 67,6 & $67(120)$ & 55,8 & $\mathrm{p}^{1}=0,0029^{*}$ \\
\hline & Agamenon Magalhães & $18(27)$ & 66,7 & $43(80)$ & 53,8 & $61(107)$ & 57,0 & $\mathrm{p}^{1}=0,2411$ \\
\hline \multirow[t]{2}{*}{ Cefepima } & $\mathrm{HC}$ & $17(49)$ & 34,7 & $43(65)$ & 66,2 & 60 (114) & 52,6 & $\mathrm{p}^{1}=0,0009^{*}$ \\
\hline & Agamenon Magalhães & 9 (23) & 39,1 & $21(73)$ & 28,8 & $30(96)$ & 31,3 & $\mathrm{p}^{1}=0,3498$ \\
\hline \multirow[t]{2}{*}{ Cefotaxima } & $\mathrm{HC}$ & $2(51)$ & 3,9 & $6(68)$ & 8,8 & $8(119)$ & 6,7 & $\mathrm{p}^{2}=0,4637$ \\
\hline & Agamenon Magalhães & $3(22)$ & 13,6 & $5(73)$ & 6,8 & $8(95)$ & 8,4 & $p^{2}=0,3816$ \\
\hline \multirow[t]{2}{*}{ Ciprofloxacina } & $\mathrm{HC}$ & $18(51)$ & 35,3 & $37(64)$ & 57,8 & 55 (115) & 47,8 & $\mathrm{p}^{1}=0,0163^{*}$ \\
\hline & Agamenon Magalhães & $12(27)$ & 44,4 & $30(83)$ & 36,1 & $42(110)$ & 38,2 & $p^{1}=0,4406$ \\
\hline \multirow[t]{2}{*}{ Gentamicina } & $\mathrm{HC}$ & $18(50)$ & 36,0 & $42(68)$ & 61,8 & 60 (118) & 50,8 & $\mathrm{p}^{1}=0,0057^{\star}$ \\
\hline & Agamenon Magalhães & $12(26)$ & 46,2 & $22(77)$ & 28,6 & 34 (103) & 33,0 & $\mathrm{p}^{1}=0,0993$ \\
\hline \multirow[t]{2}{*}{ Imipenem } & $\mathrm{HC}$ & $19(51)$ & 37,3 & $44(67)$ & 65,7 & $63(118)$ & 53,4 & $\mathrm{p}^{1}=0,0022^{*}$ \\
\hline & Agamenon Magalhães & $15(26)$ & 57,7 & $38(81)$ & 46,9 & $53(107)$ & 49,5 & $\mathrm{p}^{1}=0,3389$ \\
\hline \multirow[t]{2}{*}{ Meropenem } & $\mathrm{HC}$ & $15(31)$ & 48,4 & $22(34)$ & 64,7 & $37(65)$ & 56,9 & $\mathrm{p}^{1}=0,1845$ \\
\hline & Agamenon Magalhães & $17(26)$ & 65,4 & $38(75)$ & 50,7 & 55 (101) & 54,5 & $\mathrm{p}^{1}=0,1941$ \\
\hline \multirow{2}{*}{$\begin{array}{l}\text { Piperacilina-tazo- } \\
\text { bactam }\end{array}$} & $\mathrm{HC}$ & 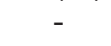 & - & & - & $=5$ & - & \\
\hline & Agamenon Magalhães & $19(27)$ & 70,4 & $52(81)$ & 64,2 & $71(108)$ & 65,7 & $p^{1}=0,5583$ \\
\hline \multirow[t]{2}{*}{ Ceftazidima } & $\mathrm{HC}$ & - & - & - & - & - & - & - \\
\hline & Agamenon Magalhães & $9(23)$ & 39,1 & $18(66)$ & 27,3 & $27(89)$ & 30,3 & $\mathrm{p}^{1}=0,2868$ \\
\hline
\end{tabular}

* Associação significativa ao nível de $5 \%$.

$\mathrm{n}=$ cepas sensíveis testadas

1 Teste Qui-quadrado de Pearson.

2 Teste Exato de Fisher.

$\mathrm{n}=$ representa o número de cepas sensíveis e os números entre parêntesis representam o total de amostras analisadas.

Tabela 3 - Percentual de Resistência Cruzada da Ciprofloxacina e Amicacina com os Antibióticos: Imipenem, Meropenem, PiperacilinaTazobactam nos Hospitais Estudados

\begin{tabular}{|c|c|c|c|c|c|c|}
\hline \multirow{3}{*}{ Antibióticos } & \multicolumn{4}{|c|}{ Hospital } & \multirow{2}{*}{\multicolumn{2}{|c|}{ Os Dois Hospitais }} \\
\hline & \multirow{2}{*}{$\begin{array}{c}\text { HC } \\
\text { Resistentes (Testadas) }\end{array}$} & \multicolumn{3}{|c|}{ Agamenon Magalhães } & & \\
\hline & & $\%$ & Resistentes (Testadas) & $\%$ & Resistentes (Testadas) & $\%$ \\
\hline \multicolumn{7}{|c|}{ Resistência cruzada a ciprofloxacina } \\
\hline Imipenem & $54(155)$ & 34,8 & $43(107)$ & 40,2 & $97(262)$ & 37 \\
\hline Meropenem & $31(88)$ & 35,2 & $41(101)$ & 40,6 & $72(189)$ & 38,1 \\
\hline Piperacilina-Tazobactam & NT & - & $32(108)$ & 29,6 & $32(108)$ & 29,6 \\
\hline \multicolumn{7}{|c|}{ Resistência cruzada à amicacina } \\
\hline Imipenem & $46(151)$ & 30,6 & $38(108)$ & 35,2 & $84(259)$ & 32,4 \\
\hline Meropenem & $27(88)$ & 30,7 & $36(101)$ & 35,6 & $63(189)$ & 33,3 \\
\hline Piperacilina-Tazobactan & NT & - & $25(109)$ & 22,9 & $25(109)$ & 22,9 \\
\hline
\end{tabular}

Os valores entre parêntesis representam o total de amostras analisadas.

NT = Não testada 
Tabela 4 - Número de Amostras Resistentes em Painel de Oito Antibióticos nos Hospitais Estudados

\begin{tabular}{|c|c|c|c|c|c|c|}
\hline \multirow{3}{*}{$\begin{array}{l}\text { Número de Fármacos com } \\
\text { Resistência }\end{array}$} & \multicolumn{4}{|c|}{ Hospitais } & \multirow{2}{*}{\multicolumn{2}{|c|}{ Os Dois Hospitais }} \\
\hline & \multicolumn{2}{|r|}{$\mathrm{HC}$} & \multicolumn{2}{|c|}{ Agamenon Magalhães } & & \\
\hline & Resistentes & $\%$ de Resistentes & Resistentes & $\%$ de Resistentes & Resistentes & $\%$ de Resistentes \\
\hline Um & 21 & 19,4 & 11 & 11,4 & 32 & 15,5 \\
\hline Dois & 14 & 12,8 & 9 & 9,3 & 23 & 11,2 \\
\hline Três & 11 & 10,1 & 16 & 16,5 & 27 & 13,1 \\
\hline Quatro & 9 & 8,2 & 13 & 13,4 & 22 & 10,7 \\
\hline Cinco & 9 & 8,2 & 8 & 8,2 & 17 & 8,2 \\
\hline Seis & 28 & 25,7 & 13 & 13,4 & 41 & 19,9 \\
\hline Sete ou mais & 17 & 15,6 & 27 & 27,8 & 44 & 21,4 \\
\hline Total & 109 & 100,0 & 97 & 100,0 & 206 & 100,0 \\
\hline
\end{tabular}

OBS: foram considerados para análise os seguintes antibióticos: amicacina, aztreonam, cefepima, ciprofloxacina, gentamicina, imipenem, meropenem, piperacilinatazobactam.

$34,8 \%$ no HC, 40,2\% no HAM e 37\% em ambos; para o meropenem $35,2 \%$ no $\mathrm{HC}, 40,6 \%$ no $\mathrm{HAM}$ e $38,1 \%$ em ambos os hospitais. No HAM, para a piperacilinatazobactam as taxas de resistência cruzada foram $29,6 \%$.

A tabela 4 mostra os resultados da sensibilidade das cepas de $P$. aeruginosa frente a um grupo de oito antimicrobianos (amicacina, aztreonam, cefepima, ciprofloxacina, gentamicina, imipenem, meropenem, piperacilina-tazobactam). Cerca de $98(32,2 \%)$ cepas não apresentou resistência a nenhum antibiótico, 32 $(10,5 \%)$ demonstraram resistência a um antibiótico; 23 (7,6\%) para dois antibióticos; 27 (8,9\%) para três antibióticos; 22 (7,2\%) para quatro antibióticos; 17 (5,6\%) para cinco antibióticos; 41 (13,5\%) para seis antibióticos e 44 (14,5\%) para sete ou mais antibióticos.

\section{DISCUSSÃO}

O presente estudo evidenciou taxas de resistência antimicrobiana da Pseudomonas aeruginosa similares ao estudo de Kiffer e col. ${ }^{7}$, que compilou cepas de 21 hospitais de várias regiões do Brasil e mais elevadas do que as descritas em outros países $5,6,11,14,26,27$. Revelou elevada prevalência de multi-resistência, com 49,7\% das cepas resistentes a três antibióticos ou mais e $28 \%$ das cepas resistentes a seis antibióticos ou mais. Adicionalmente demonstrou taxas de resistência cruzada entre os carbapenêmicos e a piperacilina-tazobactam e os fármacos comumente utilizados como adjuvantes no tratamento das infecções graves causadas pelas Pseudomonas como os aminoglicosídeos e quinolonas entre $22,9 \%$ e $38,1 \%$, refletindo dificuldade nas opções de associação de antimicrobianos para tratamentos com esquemas combinados.

Várias limitações do estudo devem ser salientadas. Por se tratar de análise retrospectiva, baseada em informações coletadas dos laboratórios, encontraram-se falhas nos registros dos dados. Não houve uniformização do número de antibióticos observados nos laboratórios dos dois hospitais que participaram do estudo (piperacilina-tazobactam e ceftazidima somente foram observadas no HAM e nem todos os antibióticos foram testados para todas as cepas). Finalmente as culturas realizadas não representam necessariamente infecção, podendo decorrer apenas de colonização. De qualquer modo, são representativas das cepas circulantes naqueles hospitais, com potencial para colonizar e, subseqüentemente causar infecção nos pacientes internados naquelas instituições.

Os dados deste estudo mostraram que o fármaco mais ativo contra a $P$. aeruginosa é a piperacilina-tazobactam, com a ressalva de que foi testada apenas no HAM. Este resultado foi semelhante ao do estudo de Pellegrino e col. realizado no Rio de Janeiro e na Argentina ${ }^{1,27}$. A seguir o aztreonam e a amicacina, esta última, posicionada como um dos antibióticos com meIhor atividade contra a $P$. aeruginosa, aspecto também documentado em outros locais como a Itália ${ }^{12}$. Com relação aos carbapenêmicos, opções tradicionais no tratamento das infecções por Pseudomonas pela elevada potência e amplo espectro, ocuparam a quarta e quinta posição, com sensibilidades baixas, ou seja, entre $57 \%$ e $58 \%$. Estudos latino-americanos recentes vêm mostrando redução significativa da ação deste grupo de antimicrobianos contra a $P$. aeruginosa em decorrência da produção de metallo-beta-lactamases na região $0^{5,19,28}$ mostrando taxas de susceptibilidade de $64 \% 7,19$, muito próximas às encontradas no presente estudo. A ciprofloxacina mostrou sensibilidade abaixo de $50 \%$, certamente reflexo do seu uso em larga escala em nível ambulatorial e hospitalar, não só no Re- 
cife, mas em todo o mundo ${ }^{11}$. Entre as cefalosporinas com ação anti-pseudomonas a cefepima apresentou sensibilidade de $32 \%$ e a ceftazidima foi efetiva contra apenas $30,5 \%$ das cepas no HAM. A tendência à menor sensibilidade às cefalosporinas tem sido descritas em outras regiões ${ }^{11,12}$, mas é muito provável, que o uso em larga escala das cefalosporinas de $3^{\mathrm{a}}$ geração como opção terapêutica quase exclusiva, por muitos anos, para pacientes graves nas emergências e enfermarias de hospitais públicos do Recife se reflita neste resultado. Assim, embora a literatura estrangeira cite as cefalosporinas anti-pseudomonas como boa opção terapêutica para o tratamento das infecções por pseudomonas, não seriam medicações de primeira escolha nestas instituições.

Apesar de controverso, o uso de esquemas combinados de antimicrobianos parece eficiente para pacientes com infecções graves por Pseudomonas, particularmente as infecções com bacteremia ${ }^{3}$. Do ponto de vista microbiológico os dados do presente estudo mostraram que a amicacina é o melhor fármaco para associação de antimicrobianos por ser o de maior sensibilidade e apresentar a menor resistência cruzada com os carbapenêmicos e B-lactâmicos. Soma-se ainda o menor potencial dos aminoglicosídeos em induzir multi-resistência bacteriana comparados às quinolonas ${ }^{29}$. Entretanto, não se pode esquecer os risco de nefrotoxicidade e ototoxicidade induzidos pelos aminoglicosídeos ${ }^{30}$ que podem resultar em lesões significativas. Por outro lado, a associação de antimicrobianos com quinolonas, apesar da sua menor toxicidade parece ser desvantajosa, pois além da elevada freqüência de resistência às quinolonas, a resistência cruzada com os carbapenêmicos e B-lactâmicos ficam em torno de $30 \%$ a $40 \%$, reduzindo o potencial terapêutico da associação.

O presente estudo evidenciou elevada prevalência de resistência a múltiplos fármacos, dado este corroborado pelos resultados do estudo de Magalhães e col. ${ }^{21}$ na cidade do Recife, cuja análise de 48 cepas de $P$. aeruginosa, demonstrou $62,5 \%$ de positividade para produção de enzima beta-lactamase, com um perfil de resistência amplo, apresentando apenas susceptibilidade à polimixina.

Observaram-se diferenças significativas no perfil de sensibilidade das Pseudomonas nas duas instituições, com maiores índices de resistência no HAM comparadas ao $\mathrm{HC}$, o que pode refletir a tradição da comissão de infecção hospitalar $(\mathrm{CClH})$ atuante no $\mathrm{HC}$, que foi uma das pioneiras no controle de antimicrobianos no
Brasil ou diferenças de clientela, com pacientes mais críticos no HAM, pois além de emergência o HAM possui maternidade e berçário de alto risco, UTI coronariana e geral, com maior número de leitos críticos do que o HC. A segunda diferença encontrada foi mais curiosa. Enquanto no $\mathrm{HC}$ existiu maior resistência e mais evidente nas cepas de Pseudomonas da UTI comparadas à das alas, no HAM a diferença de resistência entre as Pseudomonas dos dois locais não foi significativa. A explicação mais provável é a de que pacientes graves têm permanecido nas alas no HAM por um déficit crônico de vagas em UTI nas instituições públicas, ou eventualmente, que os pacientes crônicos ao receberem alta das unidades críticas permanecem por longo tempo nas enfermarias, modificando esta flora com a persistência e disseminação de cepas multiresistentes. Essas diferenças entre instituições tornam evidentes a importância de conhecer a microbiota e o perfil de resistência de cada instituição que podem diferir significativamente, mesmo quando localizadas no mesmo município.

\section{CONCLUSÃO}

Os dados apresentados mostraram taxas bastante elevadas de Pseudomonas aeruginosa resistentes a múltiplos fármacos, dificuldades nas opções de fármacos para tratamentos combinados e a necessidade de vigilância individualizada do perfil de resistência em cada instituição. Essas informações devem auxiliar na adoção de políticas concretas de utilização racional dos antimicrobianos e de redução da disseminação das cepas resistentes nas instituições.

\section{REFERÊNCIAS}

01. Pellegrino FL, Teixeira LM, Carvalho Md Mda G et al - Occurrence of a multidrug-resistant Pseudomonas aeruginosa clone in different hospitals in Rio de Janeiro, Brazil. J Clin Microbiol, 2002;40:2420-2424.

02. Osmon S, Ward S, Fraser VJ et al - Hospital mortality for patients with bacteremia due to Staphylococcus aureus or Pseudomonas aeruginosa. Chest, 2004;125:607-616.

03. Safdar N, Handelsman J, Maki DG - Does combination antimicrobial therapy reduce mortality in Gram-negative bacteraemia? A meta-analysis. Lancet Infect Dis, 2004;4:519-527.

04. Sader HS, Gales AC, Pfaller MA et al - Pathogen frequency and resistance patterns in Brazilian hospitals: summary of results from three years of the SENTRY Antimicrobial Surveillance Program. Braz J Infect Dis, 2001;5:200-214.

05. Andrade SS, Jones RN, Gales AC et al - Increasing prevalence of antimicrobial resistance among Pseudomonas aeruginosa isolates in Latin American medical centres: 5 year report of the SENTRY Antimicrobial Surveillance Program (1997-2001). J Antimicrob Chemother, 2003;52:140-141.

06. Gales AC, Sader H HS, Jones RN - Respiratory tract pathogens isolated from patients hospitalized with suspected pneumonia in Latin America: 
frequency of occurrence and antimicrobial susceptibility profile: results from the SENTRY Antimicrobial Surveillance Program (1997-2000). Diagn Microbiol Infect Dis, 2002;44:301-311.

07. Kiffer C, Hsiung A, Oplustil C et al - Antimicrobial susceptibility of Gramnegative bacteria in Brazilian hospitals: the MYSTYC Program Brazil 2003. Braz J Infect Dis, 2005;9:216-224.

08. Van Eldere $\mathrm{J}$ - Multicentre surveillance of Pseudomonas aeruginosa susceptibility patterns in nosocomial infections. J Antimicrob Chemother, 2003;51:347-352.

09. Friedland I, Stinson L, Ikaiddi M et al - Phenotypic antimicrobial resistance patterns in Pseudomonas aeruginosa and Acinetobacter: results of a Multicenter Intensive Care Unit Surveillance, 1995-2000. Diagn Microbiol Infect Dis, 2003;45:245-250.

10. Bayram A, Balci I - Patterns of antimicrobial resistance in a surgical intensive care unit of a university hospital in Turkey. BMC Infect Dis, 2006;6:155.

11. McGowan JE Jr - Resistance in nonfermenting gram-negative bacteria: multidrug resistance to the maximum. Am J Infect Control, 2006;34:(Suppl1):S29-S37.

12. Nicoletti G, Schito G, Fadda G et al - Bacterial isolates from severe infections and their antibiotic susceptibility patterns in Italy: a nationwide study in the hospital setting. J Chemother, 2006;18:589-602.

13. Landman D, Bratu S, Kochar S et al - Evolution and antimicrobial resistance among Pseudomonas aeruginosa, Acinetobacter baumannii and Klebsiella pneumoniae in Brooklyn, NY. J Antimicrob Chemother, 2007;60:78-82.

14. Raja NS, Singh NN - Antimicrobial susceptibility pattern of clinical isolates of Pseudomonas aeruginosa in a tertiary care hospital. J Microbiol Immunol Infect, 2007;40:45-49.

15. Li XZ, Zhang L, Poole K - Interplay between the MexA-MexB-OprM multidrug efflux system and the outer membrane barrier in the multiple antibiotic resistance of Pseudomonas aeruginosa. J Antimicrob Chemother, 2000;45:433-436.

16. Cavallo JD, Plesiat P, Couetdic G et al - Mechanisms of beta-lactam resistance in Pseudomonas aeruginosa: prevalence of OprM-overproducing strains in a French multicentre study (1997). J Antimicrob Chemother, 2002;50:1039-1043.

17. Livermore DM - Multiple mechanisms of antimicrobial resistance in Pseudo- monas aeruginosa: our worst nightmare? Clin Infect Dis, 2002;234:634-640.

18. Jacoby GA, Munoz-Price LS - The new beta-lactamases. N Engl J Med, 2005;352:380-391.

19. Sader HS, Castanheira M, Mendes RE et al - Dissemination and diversity of metallo-beta-lactamases in Latin America: report from the SENTRY Antimicrobial Surveillance Program. Int J Antimicrob Agents, 2005;25:57-61.

20. Lepper PM, Grusa E, Reichl H et al - Consumption of imipenem correlates with beta-lactam resistance in Pseudomonas aeruginosa. Antimicrob Agents Chemother, 2002;46:2920-2925.

21. Magalhaes V, Lins AK, Magalhaes M - Mettalo-beta-lactamase producing Pseudomonas aeruginosa strains isolated in hospitals in Recife, PE, Brazil. Braz J Microbiol, 2005;36:123-125.

22. National Committee for Clinical Laboratory Standards. Performance standards for disk susceptibility tests; seventh edition. Approved Standard M2-A7.NCCLS, Wayne, PA, 2000.

23. National Committee for Clinical Laboratory Standards. Performance standards for antimicrobial susceptibility testing; twelfth Informational Supplement. M100-S12.NCCLS, Wayne, PA. 2002.

24. Chapman ADG - Practical Statistics for Medical Research, $1^{\text {st }}$ Ed, London: CRC press, 1991.

25. Zar JH - Biostatistical Analysis, $4^{\text {th }}$ Ed, New Jersey: Prentice-Hall, 1999.

26. Kato K, Iwai S, Kumasaka K et al - Survey of antibiotic resistance in Pseudomonas aeruginosa by the Tokyo Johoku Association of Pseudomonas Studies. J Infect Chemother, 2001;7:258-262.

27. Casellas JM, Tome G, Bantar C et al - Argentinean collaborative multicenter study on the in vitro comparative activity of piperacillin-tazobactam against selected bacterial isolates recovered from hospitalized patients. Diagn Microbiol Infect Dis, 2003;47:527-537.

28. Gales AC, Torres PL, Vilarinho DS et al - Carbapenem-resistant Pseudomonas aeruginosa outbreak in an intensive care unit of a teaching hospital. Braz J Infect Dis, 2004;8:267-271.

29. Nseir S, Di Pompeo C, Soubrier S et al - First-generation fluoroquinolone use and subsequent emergence of multiple drug-resistant bacteria in the intensive care unit. Crit Care Med, 2005;33:283-289.

30. Gilbert DN - Aminoglycosides, em: Mandell GL, Bennett JE, Dolin R Principles and Practice of Infectious Diseases. Philadelphia, $6^{\text {th }} \mathrm{Ed}$,: Elsevier, 2005;328-355. 